\title{
Synthesis of allyl e alkylarenes by Barbier alkylation of diazonium salts in aqueous medium
}

\author{
Idália Estevam $^{\mathrm{a}^{\star}}$, Emmanuelle Silva ${ }^{\mathrm{a}}$, Lothar Bieber ${ }^{\mathrm{b}}$
}

${ }^{a}$ Departamento de Ciências Exatas e da Terra, Universidade do Estado da Bahia, Salvador-BA ; ${ }^{b}$ Departamento de Química Fundamental, Universidade Federal de Pernambuco, Recife-PE, Brazil

*iestevam@uneb.br

Keywords: Barbier alkylation, diazonium salts, Barbier allylation

\section{INTRODUCTION}

Barbier reactions in aqueous media are mainly performed with allylic halides and carbonyl substrates. This paper presents new a methodology for allylation of ionic substrates in aqueous such as diazonium salts. Getting the first product of allylation as well as of alkylation with halides saturated.

Some allylbenzenes are obtained from sassafras oil extraction. However the propenylbenzene is not obtained from natural sources. During the studies on the allylation of diazoniun salts in water, the propenylbenzene was obtained as a major product in good yields. Due to the good result, we examined the applicability of the method to other saturated halides and different anilines.

\section{RESULTS AND DISCUSSION}

Benzene diazonium salt (2), generated from nitrous acid and aniline (1), was reacted with allylbromide. The reactional media was neutralized by calcium carbonate addition. None co-solvents or catalysts improved the results of the reaction. Applying the method for isopropyl iodide, using the DMSO: $\mathrm{H}_{2} \mathrm{O}$ (9:1) mixture, the yielding was 54\%. Examining the reaction with the 2-methylaniline, 2-isopropyltoluene (3) was formed with $61 \%$ of yielding.

Figure 1. Insert the figure caption here

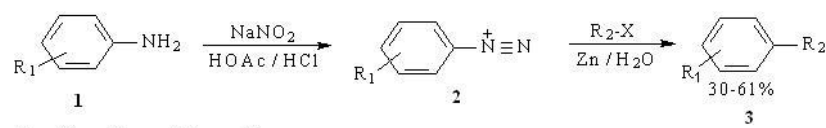

$\mathrm{R}_{1}=\mathrm{H} ; 0-\mathrm{Me} ; \mathrm{p}-\mathrm{NO}_{2} ; \mathrm{p}-\mathrm{F}$

$\mathrm{R}_{2}-\mathrm{X}=$ allyl-Br, i-Pr-I; $\mathrm{t}-\mathrm{B}$ ut $-\mathrm{Br}$

Table 1. Reaction medium

\begin{tabular}{|l|l|l|l|}
\hline Entry & Solvent & $\mathrm{R}_{1} / \mathrm{R}_{2}$ & Yield (\%) \\
\hline 1 & $\mathrm{H}_{2} \mathrm{O} / \mathrm{HCl}$ & $\mathrm{H} /$ allyl & 56 \\
\hline 2 & $\mathrm{H}_{2} \mathrm{O} / \mathrm{HOAc}^{1}$ & $\mathrm{H} /$ allyl & 16 \\
\hline 3 & $\mathrm{H}_{2} \mathrm{O} / \mathrm{HOAc}^{2} / \mathrm{HCl}$ & $\mathrm{H} /$ allyl & 45 \\
\hline
\end{tabular}

${ }^{*} 2 \mathrm{mmol}$ of acetic acid

${ }^{2} \mathrm{HOAC}(\mathrm{aq}) 50 \% \mathrm{w} / \mathrm{w}-2 \mathrm{mmol}$ of $\mathrm{HCl}$
Table 2. Alkylarene synthesis

\begin{tabular}{|l|l|l|l|}
\hline Entry & $\mathrm{R}_{1} / \mathrm{R}_{2}$ & solvent & Yield \\
\hline 1 & $\mathrm{H} /$ allyl & $\mathrm{H}_{2} \mathrm{O}$ & 56 \\
\hline 2 & $\mathrm{H} / \mathrm{i}-\mathrm{Pr}$ & DMSO $: \mathrm{H}_{2} \mathrm{O}^{1}$ & 54 \\
\hline 3 & $2-\mathrm{CH}_{3} /$ allyl & $\mathrm{H}_{2} \mathrm{O}$ & 54 \\
\hline 4 & $4-\mathrm{F} /$ allyl & $\mathrm{H}_{2} \mathrm{O}$ & 34 \\
\hline 5 & $4-\mathrm{F} / \mathrm{i}-\mathrm{Pr}$ & DMSO: $\mathrm{H}_{2} \mathrm{O}^{1}$ & 61 \\
\hline 6 & $2-\mathrm{CH}_{3} / \mathrm{i}-\mathrm{Pr}$ & DMSO: $\mathrm{H}_{2} \mathrm{O}^{1}$ & 61 \\
\hline
\end{tabular}

\section{CONCLUSION}

We have investigated and presented a valuable alternative for one-pot alkylarenes synthesis. Our method proceeds under aqueous conditions, utilizes readily available, inexpensive, amines. Moreover, our one-pot alkylation with isopropyl halides is very innovative.This makes a method economical and regiosseletive.

\section{ACKNOWLEDGEMENTS}

FAPESB; UNEB; UFPE Analytical Center

\section{REFERENCES}

1 a) Anastas, P. T.; Williamson, T. C. Green Chemistry; Oxford University Press: New York, 1998; b) Afonso, C. A. M.; Crespo, J. G. Green Separation Processes.; WILEY-VCH Verlag GmbH \& Co. KGaA, Weinheim, 2005

2 Li, C.J. Chem. Rev. 2005, 105, 3095

${ }^{3}$ Costa, P. R. R. Quim. Nova 2000, 23, 357

${ }^{4}$ Tsukinoki, T., Tsuzuki, H Green Chemistry, 2001, 3, 37. 